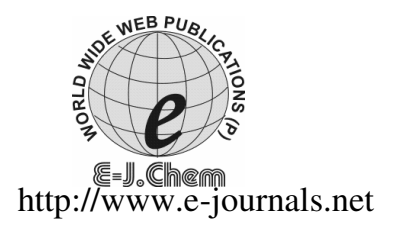

\author{
ISSN: 0973-4945; CODEN ECJHAO \\ E-Journal of Chemistry \\ $2011,8(2), 748-752$
}

\title{
Synthesis of Some New Benzimidazole Derivatives of Pharmaceutical Interest
}

\author{
FAWZIA ZAKARIA EL-ABLACK \\ Chemistry Department, Damietta Faculty of Science \\ Mansoura University, Egypt \\ fawzia@mans.edu.eg
}

Received 27 March 2010; Accepted 20 May 2010

\begin{abstract}
Reaction of 2-(aminomethyl)benzimidazole dihydrochloride (1) with ethyl acetoacetate was studied to give diazepinone-benzimidazole derivative (2), while, treatment of $\mathbf{1}$ with phenylhydrazono ethylacetoacetate afforded phenylhydrazino diazepinone derivative (3). On the other hand, reaction of $\mathbf{1}$ with acetyl acetone resulted in the formation of diazepine derivative (4). The reaction of $\mathbf{1}$ with ethyl cyanoacetate was studied to give 3aminodiazepinone derivative (5). Also the reaction of $\mathbf{1}$ with acetophenone and/or benzophenone has been investigated to give the fused imidazolines $\mathbf{6}$ and 7 respectively, while the reaction of $\mathbf{1}$ with cyclopentanone gave benzimidazolyl derivative (8). Treatment of $\mathbf{1}$ with chloroacetyl chloride gave the fused pyrazinone (9). The treatment of $\mathbf{1}$ with benzoin gave the derivative (10). The structures of the hitherto unknown compounds have been confirmed from analytical and spectral data. The newly synthesized compounds were screened for antibacterial and antifungal activity
\end{abstract}

Keywords Diazepino-Benzimidazole, Pyrazino -benzimidazole and midazolo $\{1.5$-a $\}$ benzimidazole, Antibacterial, Antifungal activity

\section{Introduction}

Benzimidazole derivatives have been reported to have a wide range of pharmacological and biochemical activity; It consider to be CNS depressant, anti-Parkinson, antiviral activity, anti ulcerative, antihypertensive, antifungal, antitumor, antihistaminic, anti-bacterial and antihelminthes agents ${ }^{1-4}$.

Following the above findings and continuous to our previous work ${ }^{5-8}$ directed towards the synthesis of fused heterocyclic compounds of potential biological activities the utilizing of 2 -aminomethyl benzimidazole ${ }^{9}$ as a title compound for the synthesis of some new benzimidazole derivatives was studied. 
Due to the wide range of pharmacological activity and synthetic application of benzimidazoles in this study it was planned to synthesize benzimidazole derivatives using 2-aminomethyl benzimidazole ${ }^{9}$ (1). Thus the reaction of 2-aminomethyl benzimidazole (1) with ethyl acetoacetate gave diazepinone derivative (2). IR spectrum revealed the disappearance of $\mathrm{NH}_{2}$ absorption band at $3400-3550 \mathrm{~cm}^{-1}$ and the appearance of absorption band at $1680 \mathrm{~cm}^{-1} \mathrm{CO}$ group. NMR spectra established the presence of a signals at $\delta 3.78\left(\mathrm{~s}, 2 \mathrm{H}, \mathrm{CH}_{2}\right)$ and at $\delta 2.6\left(\mathrm{~s}, 2 \mathrm{H}, \mathrm{CH}_{2}\right)$ so it not present as tautomeric enaminone structure (2). Treatment of 1 with phenylhydrazeno ethyl acetoacetate afforded (1,4-diazepino [1,2-a] benzimidazole derivative (3), the structure of 3 was based on the analytical and spectral data, the ${ }^{1} \mathrm{H}$ NMR spectrum reveal the presence of a signal at $\delta 8.12(\mathrm{~s}, 1 \mathrm{H}, \mathrm{NH})$ and $\delta 2.89\left(\mathrm{~s}, 2 \mathrm{H}, \mathrm{CH}_{2}\right)$. While the treatment of $\mathbf{1}$ with acetyl acetone in $1 \mathrm{~N}$ sodium hydroxide solution resulted in the formation of 3,5-dimethyl-[1,3]diazipene[1,2-a]benzimidazole derivative (4), the structure of $\mathbf{4}$ was inferred from the obtained analytical and spectral data. IR spectrum revealed the disappearance of $\mathrm{NH}_{2}$ absorption band and the presence of absorption band at $1625 \mathrm{~cm}^{-1}$ for $\mathrm{C}=\mathrm{N}$.

When 1 was treated with ethyl cyanoacetate in ethanol containing a catalytic amount of piperdene gave 3-amino diazepinone derivative (5). The structure of $\mathbf{5}$ was based on the analytical and spectral data. IR spectrum reveal the presence of $\left(\mathrm{NH}_{2}\right)$ at $3345 \mathrm{~cm}^{-1}$, the ${ }^{1} \mathrm{H}$ NMR spectrum revealed the presence of signal at $\delta 6.5\left(\mathrm{~s}, 2 \mathrm{H}, \mathrm{NH}_{2}\right), \delta 7.2-7.7(\mathrm{~m}, 4 \mathrm{H}$, $\mathrm{ArH}), \quad \delta 2.73\left(\mathrm{~s}, 2 \mathrm{H}, \mathrm{CH}_{2}\right)$ and $\delta 2.85\left(\mathrm{~s}, 2 \mathrm{H}, \mathrm{CH}_{2}\right)$ so it not present in its tautomeric enaminone structure $(\mathbf{5})$. .

The reaction of $\mathbf{1}$ with acetophenone and benzophenone resulted in the formation of $1 H$-imidazol[1,5-a]benzimidazole derivatives $(\mathbf{6 , 7})$ respectively. The structure of 6 was confirmed from the obtained analytical and spectral data. IR spectrum of 6 displayed absorption band at $3330 \mathrm{~cm}^{-1}(\mathrm{NH}),{ }^{1} \mathrm{H}$ NMR spectrum displayed signals at $\delta 2.1(\mathrm{~s}, 1 \mathrm{H}, \mathrm{NH})$ (Table 3$)$.

As a further extension of the above studies, the reaction of $\mathbf{1}$ with cyclopentanone gave benzimidazole derivative $(\mathbf{8})$. The structure of $\mathbf{8}$ was assigned from the analytical and spectral data. ${ }^{1} \mathrm{H}$ NMR spectrum displayed signal at $\delta 2.3(\mathrm{~s}, 1 \mathrm{H}, \mathrm{NH})$.

Treatment of 1 with chloroacetyl chloride gave pyrazino\{1,2-a\}benzimidazol-3(4H)one derivative (9). The structure of $\mathbf{9}$ was established from the obtained analytical and spectral data. IR spectrum revealed absorption band at $3335 \mathrm{~cm}^{-1} \mathrm{NH}$. On the other hand, the reaction of 1 with benzoin in ethanol containing catalytic amount of piperdene gave 3, 4diphenyl-1,4-dihydropyrazino[1,2-a]benzimidazole (10). ${ }^{1} \mathrm{H}$ NMR revealed signal at $\delta 5.01$ (s, H, CH olefinic) and at $\delta 4.78\left(\mathrm{~s}, 2 \mathrm{H}, \mathrm{CH}_{2}\right)$ (Scheme 1\& 2).

\section{Experimental}

Melting points (uncorrected) were determined on Fisher-Johns melting point apparatus. Elemental analysis was performed in the microanalysis unit, Cairo University. IR spectra were recorded by means of pressed $\mathrm{KBr}$ on a Perkin-Elmer 883 infrared spectrophotometer. ${ }^{1} \mathrm{H}$ NMR spectra were recorded on a Varian $90 \mathrm{MHz}$ in $\mathrm{CDCl}_{3}$. 
<smiles>CC1=CC(=O)C(C)=CC(=O)n2c(nc3ccccc32)C(CC(C)C)CN1</smiles>

(2)

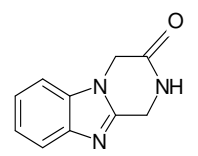

(9)

(9)<smiles>[CH]COC(=O)Cl</smiles>

Cl-CO- $\mathrm{CH}_{2}-\mathrm{Cl}$ cyclopentanone

(8)

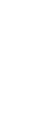

benzophenone
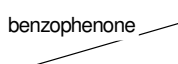<smiles>[H][R14]1CCCCC1</smiles><smiles>NCc1nc2ccccc2[nH]1</smiles>

(1)<smiles>NC1=NCc2nc3ccccc3n2C(=O)C1</smiles>

(4)

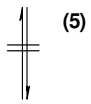<smiles>NC1=CC(=O)n2c(nc3ccccc32)CN1</smiles>

(5')

\section{Scheme 1}<smiles>NCc1nc2ccccc2[nH]1</smiles>

(1)

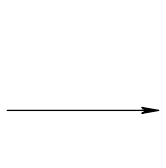

Scheme 2

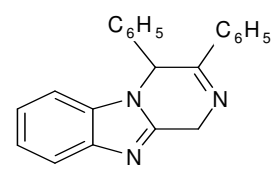

(10)

Formation of 3-methyl-1,4-dihydro-5H\{1,4\}diazepino\{1,2-a\}benzimidazol-5-one (2) To a solution of $1(0.01 \mathrm{~mol})$ in $\mathrm{NaOH}$ solution $(1 \mathrm{~N}, 25 \mathrm{~mL})$ ethyl acetoacetate $(0.01 \mathrm{~mol})$ was added. The reaction mixture was heated on water bath for $3 \mathrm{~h}$, left to cool and the solid product was separated, filtered off and recrystallized from the appropriate solvent ( Table 1).

Formation of (4E)-3-methyl-4-(2-phenylhydrazinylidene)-1,4-dihydro-5H-[1,4]diazepino [1,2-a]benzimidzol-5-one (3)

To a 0.01 mol solution of $\mathbf{1}$ in $\mathrm{NaOH}$ solution $(1 \mathrm{~N}, 25 \mathrm{~mL})$, phenylhydrazino ethyl acetoacetate $(0.01 \mathrm{~mol})$ was added. The reaction mixture was heated on water-bath with continuous stirring and cooled. The obtained product was filtered off and recrystallized from the proper solvent (Table 1). 
Formation of 3,5-dimethyl-1H-\{1,4\}diazepino\{1,2-a\}benzimidazole (4)

A mixture of $1(0.01 \mathrm{~mol})$ and acetyl acetone $(0.01 \mathrm{~mol})$ in $30 \mathrm{~mL}$ ethanol containing a catalytic amount of piperidene $(1 \mathrm{~mL})$ was heated under reflux for 3-4 h. The solid product was cooled, filtered off and recrystallized from ethanol (Table 1).

Formation of 3-amino-1,4-dihydro-5H\{1,4\}diazepino\{1,2-a\}benzimidazol-5-one (5)

To a solution of $\mathbf{1}(0.01$ mole) in ethanol $(30 \mathrm{~mL})$ containing a catalytic amount of piperidene $(0.5 \mathrm{~mL})$, ethyl cyanoacetate $(0.01 \mathrm{~mole})$ was added. The reaction mixture was heated to reflux for $4 \mathrm{~h}$, cooled, poured onto ice-cold water. The obtained products were filtered off and crystallized from ethanol (Table 1).

Formation of 1-methyl-1-phenyl-2,3-dihydro-1H-imidazol[1,5-a]benzimidazole (6), and 1,1-diphenyl-2,3-dihydro-1H-imidazol[1,5-a]benzimidazole (7)

A mixture of $1(0.01 \mathrm{~mol})$ and acetophenone and/or phenzophenone $(0.01 \mathrm{~mol})$ in ethanol $(30 \mathrm{~mL})$ containing a catalytic amount of piperidene $(1 \mathrm{~mL})$ was heated to reflux for $3-4 \mathrm{~h}$, cooled and poured onto ice-cold water then acidified with dil $\mathrm{HCl}$. The solid product that separated was filtered off and crystallized from the appropriate solvent (Table 1).

Formation of $₫, \lambda$-dihydrospiro[ cyclopentane-1,5-imidazo[1,5a] benzimidazole] (8)

A mixture of $1(0.005 \mathrm{~mol})$ and cyclopentanone $(0.005 \mathrm{~mol})$ was heated under reflux for $3-4 \mathrm{~h}$ in ethanol $(30 \mathrm{~mL})$ containing piperidene $(1 \mathrm{~mL})$. The reaction mixture was cooled, poured onto acidified ice-cold water. The product was filtered off and recrystallized from the proper solvent (Table 1).

Formation of 1,2-dihydro pyrazino\{1,2-a\}benzimidazol-3(4H)-one (9)

A mixture of $1(0.01 \mathrm{~mol})$ and chloroacetyl chloride $(0.03 \mathrm{~mol})$ was heated in water bath for $3 \mathrm{~h}$, the solid product was cooled, filtered off, washed with ethanol and recrystallized from the proper solvent (Table 1).

Formation of 3,4-diphenyl-1,4-dihydropyrazino[1,2-a]benzimidazole (10)

A mixture of $1(0.01 \mathrm{~mol})$ and benzoin $(0.01 \mathrm{~mol})$ in $50 \mathrm{~mL}$ ethanol containing a catalytic amount of piperdine $(1 \mathrm{~mL})$ was heated under reflux for $4 \mathrm{~h}$, cooled, poured into ice-cooled water and acidified. The precipitated was filtered off and recrystallized from ethanol (Table 1).

Table 1. Characterization data of the compounds

\begin{tabular}{|c|c|c|c|c|c|c|c|c|}
\hline \multirow{2}{*}{ Compound } & \multirow{2}{*}{$\underset{\%}{\text { Yield, }}$} & \multirow{2}{*}{$\underset{{ }^{\circ} \mathrm{C}}{\text { M.P }}$} & \multirow{2}{*}{ Colour } & \multirow{2}{*}{ Solvent } & \multirow{2}{*}{$\begin{array}{l}\text { Formula } \\
\text { (M.Wt). }\end{array}$} & \multicolumn{3}{|c|}{ Calc./Found } \\
\hline & & & & & & $\% \mathrm{C}$ & $\% \mathrm{H}$ & $\% \mathrm{~N}$ \\
\hline \multirow[t]{2}{*}{2} & 92 & 170 & Dark & EtOH/ & $\mathrm{C}_{12} \mathrm{H}_{11} \mathrm{~N}_{3} \mathrm{O}$ & 67.59 & 5.2 & 19.71 \\
\hline & & & brown & $\mathrm{AcOH}$ & $(213.23)$ & 67.01 & 5.0 & 19.51 \\
\hline \multirow[t]{2}{*}{3} & 77.77 & 145 & brown & $\mathrm{EtOH}$ & $\mathrm{C}_{18} \mathrm{H}_{15} \mathrm{~N}_{5} \mathrm{O}$ & 68.12 & 4.76 & 22.07 \\
\hline & & & & & (317.34) & 67.99 & 4.52 & 21.96 \\
\hline \multirow[t]{2}{*}{4} & 60 & 197 & Pale & $\mathrm{EtOH}$ & $\mathrm{C}_{13} \mathrm{H}_{13} \mathrm{~N}_{3}$ & 73.91 & 6.20 & 19.89 \\
\hline & & & brown & & $(211.25)$ & 73.54 & 6.0 & 18.94 \\
\hline \multirow[t]{2}{*}{5} & 89.2 & 178 & Brown & $\mathrm{EtOH}$ & $\mathrm{C}_{11} \mathrm{H}_{10} \mathrm{~N}_{4} \mathrm{O}$ & 61.67 & 4.71 & 26.16 \\
\hline & & & & & $(214.22)$ & 61.15 & 4.25 & 25.45 \\
\hline \multirow[t]{2}{*}{6} & 65.9 & 202 & Reddish & EtOH & $\mathrm{C}_{16} \mathrm{H}_{15} \mathrm{~N}_{3}$ & 77.1 & 6.06 & 16.86 \\
\hline & & & brown & & $(249.3)$ & 76.09 & 5.83 & 16.24 \\
\hline \multirow[t]{2}{*}{7} & 89.7 & 245 & White & $\mathrm{EtOH}$ & $\mathrm{C}_{21} \mathrm{H}_{17} \mathrm{~N}_{3}$ & 81.0 & 5.50 & 13.5 \\
\hline & & & & & $(311.37)$ & 80.54 & 5.12 & 12.93 \\
\hline \multirow[t]{2}{*}{8} & 73.9 & 145 & Brown & $\mathrm{EtOH}$ & $\mathrm{C}_{13} \mathrm{H}_{15} \mathrm{~N}_{3}$ & 73.21 & 7.1 & 19.7 \\
\hline & & & & & $(213.27)$ & 72.95 & 6.85 & 19.54 \\
\hline \multirow[t]{2}{*}{9} & 63.04 & 180 & White & $\mathrm{EtOH}$ & $\mathrm{C}_{10} \mathrm{H}_{9} \mathrm{~N}_{3} \mathrm{O}$ & 64.16 & 4.85 & 22.45 \\
\hline & & & & & (187.192) & 63.95 & 4.52 & 22.01 \\
\hline \multirow[t]{2}{*}{10} & 70.2 & 120 & Yellow & $\mathrm{EtOH}$ & $\mathrm{C}_{22} \mathrm{H}_{17} \mathrm{~N}_{3}$ & 81.73 & 5.26 & 13.00 \\
\hline & & & & & 323.390 & 81.01 & 4.96 & 12.80 \\
\hline
\end{tabular}




\section{Pharmacology}

A solution of the tested compounds in acetone $(1 \mathrm{mg} / \mathrm{mL}$ ) was added to the nutrient agar (for bacteria) or medium (for fungi) mixed well and poured on plates, the tested organism was then streaked on the surface of agar or medium was incubated at $37{ }^{\circ} \mathrm{C}$ for $24 \mathrm{~h}$ (bacteria) and $28{ }^{\circ} \mathrm{C}$ for $48 \mathrm{~h}$ (fungi) and the growth was recorded the organism used were $S$. Aurous, S. Albus, E. Coli in addition to yeast (Table 2).

Table 2. In vitro biological activity of the compounds $(2,6,7,8,9)$

\begin{tabular}{cccccc}
\hline Compound & S. Albus & S.aureus & E.coli & B.subtities & Yeast \\
\hline $\mathbf{2}$ & - & 50 & - & 50 & - \\
$\mathbf{6}$ & - & 100 & - & 50 & 50 \\
$\mathbf{7}$ & 100 & 100 & 100 & - & 100 \\
$\mathbf{8}$ & 50 & - & 100 & - & 50 \\
$\mathbf{9}$ & 100 & 100 & 100 & 50 & 100 \\
\hline
\end{tabular}

Table 3. Spectral data of synthesized compounds

\begin{tabular}{|c|c|c|}
\hline Compd & IR. $\mathrm{cm}^{-1}(\mathrm{KBr})$ & ${ }^{1} \mathrm{H}$ NMR. $\delta(\mathrm{ppm})$ \\
\hline 2 & $\begin{array}{l}\text { 3095(CH aromatic), } 2965\left(\mathrm{CH}_{3}\right), 2890 \\
\left(\mathrm{CH}_{2}\right), 1675(\mathrm{C}=\mathrm{O}), 1620(\mathrm{C}=\mathrm{N}) .\end{array}$ & $\begin{array}{l}1.05\left(\mathrm{~s}, 3 \mathrm{H} . \mathrm{CH}_{3}\right) ; 3.78\left(\mathrm{~s}, 2 \mathrm{H} . \mathrm{CH}_{2}\right) ; 2.6(\mathrm{~s}, \\
\left.2 \mathrm{H} . \mathrm{CH}_{2}\right) .7 .24(\mathrm{~m}, 4 \mathrm{H}, \mathrm{ArH}) .\end{array}$ \\
\hline 3 & $\begin{array}{l}3410(\mathrm{NH}) ; 2995(\mathrm{CH} \text { aromatic }) ; 2885\left(\mathrm{CH}_{2}\right) \\
2930\left(\mathrm{CH}_{3}\right) ; 1680(\mathrm{C}=\mathrm{O}) ; 1620(\mathrm{C}=\mathrm{N}) .\end{array}$ & $\begin{array}{l}2.03\left(\mathrm{~s}, 3 \mathrm{H} . \mathrm{CH}_{3}\right) ; 2.89\left(\mathrm{~s}, 2 \mathrm{H} . \mathrm{CH}_{2}\right) ; 7.43- \\
7.65(\mathrm{~m}, 9 \mathrm{H}, \mathrm{ArH}), 8.81(\mathrm{~s}, \mathrm{H}, \mathrm{NH})\end{array}$ \\
\hline 4 & $\begin{array}{l}3042(\mathrm{CH} \text { aromatic }) ; 2965-2980\left(\mathrm{CH}_{3}\right) \\
2890\left(\mathrm{CH}_{2}\right) ; 1625(\mathrm{C}=\mathrm{N}) ; 1610(\mathrm{C}=\mathrm{C})\end{array}$ & $\begin{array}{l}1.13\left(\mathrm{~s}, 6 \mathrm{H} . \mathrm{CH}_{3}\right) ; 2.65\left(\mathrm{~s}, 2 \mathrm{H} . \mathrm{CH}_{2}\right) ; 7.1-7.7 \\
(\mathrm{~m}, 4 \mathrm{H}, \mathrm{ArH})) ; 5.1(\mathrm{~s}, \mathrm{H}, \mathrm{CH} \text { olefinic })\end{array}$ \\
\hline 5 & $\begin{array}{l}3050(\mathrm{CH} \text { aromatic }) ; 3345\left(\mathrm{NH}_{2}\right) ; 2895 \\
\left(\mathrm{CH}_{2}\right) ; 1680(\mathrm{C}=\mathrm{O}) ; 1628(\mathrm{C}=\mathrm{N}) .\end{array}$ & $\begin{array}{l}\left.\text { 2.4(s, } 2 \mathrm{H} . \mathrm{CH}_{2}\right) ; 2.8\left(\mathrm{~s}, 2 \mathrm{H}, \mathrm{CH}_{2}\right) 7.1-7.7(\mathrm{~m} \\
\text { 4H. } \mathrm{ArH}) ; 6.5\left(\mathrm{~s}, 2 \mathrm{H}, \mathrm{NH}_{2}\right)\end{array}$ \\
\hline 6 & $\begin{array}{l}3330(\mathrm{NH}) ; 3095(\mathrm{CH} \text { aromatic }) ; 2930\left(\mathrm{CH}_{3}\right) ; \\
2896\left(\mathrm{CH}_{2}\right) ; 1626(\mathrm{C}=\mathrm{N}) ; 1200-1250(\mathrm{C}-\mathrm{N}-\mathrm{C}) .\end{array}$ & $\begin{array}{l}\left.\text { 2.03(s, 3H. CH })_{3}\right) ; 3.8\left(\mathrm{~s}, 2 \mathrm{H} . \mathrm{CH}_{2}\right) ; 7.1-7.6 \\
(\mathrm{~m}, 9 \mathrm{H} . \mathrm{ArH}) ; 2.1(\mathrm{~s}, 1 \mathrm{H}, \mathrm{NH})\end{array}$ \\
\hline 7 & $\begin{array}{l}3415(\mathrm{NH}) ; 3096(\mathrm{CH} \text { aromatic }) ; 2875 \\
\left(\mathrm{CH}_{2}\right) ; 1620(\mathrm{C}=\mathrm{N})\end{array}$ & $\begin{array}{l}7.12-7.67(\mathrm{~m}, 14 \mathrm{H}, \mathrm{ArH}) ; 3.8\left(\mathrm{~s}, 2 \mathrm{H}, \mathrm{CH}_{2}\right) ; \\
2(\mathrm{~s}, 1 \mathrm{H}, \mathrm{NH}) .\end{array}$ \\
\hline 8 & $\begin{array}{l}3445(\mathrm{NH}), 3095(\mathrm{CH} \text { Aromatic), } 2870 \\
\left(\mathrm{CH}_{2}\right), 1624(\mathrm{C}=\mathrm{N})\end{array}$ & $\begin{array}{l}7.23-7.63(\mathrm{~m}, 4 \mathrm{H}, \mathrm{ArH}) ; 3.43\left(\mathrm{~s}, 2 \mathrm{H}, \mathrm{CH}_{2}\right) ; \\
2.3(\mathrm{~s}, 1 \mathrm{H}, \mathrm{NH}) ; 1.56-2.2\left(\mathrm{CH}_{2} \text { cyclopetane }\right)\end{array}$ \\
\hline 9 & $\begin{array}{l}3335(\mathrm{NH}) ; 3045(\mathrm{CH} \text { aromatic }) ; 2899\left(\mathrm{CH}_{3}\right) ; \\
2880\left(\mathrm{CH}_{2}\right) ; 1680(\mathrm{C}=\mathrm{O}) ; 1620(\mathrm{C}=\mathrm{N}) .\end{array}$ & $\begin{array}{l}3.4\left(\mathrm{~S}, 2 \mathrm{H}, 2 \mathrm{CH}_{2}\right) ; 4.46\left(\mathrm{~S}, 2 \mathrm{H}, \mathrm{CH}_{2}\right) ; 7.26- \\
7.65(\mathrm{~m}, 4 \mathrm{H}, \mathrm{ArH}) ; 8.15(\mathrm{~S}, 1 \mathrm{H}, \mathrm{NH})\end{array}$ \\
\hline 10 & $\begin{array}{l}3100(\mathrm{ArH}), 2910\left(\mathrm{CH}_{2}\right), 3085(=\mathrm{C}-\mathrm{H}) \\
1628(\mathrm{C}=\mathrm{N})\end{array}$ & $\begin{array}{l}\text { 7.06-7.62 ( m, 14H, ArH), } 5.01(\mathrm{~s}, 1 \mathrm{H}, \mathrm{CH} \\
\text { olefinic), } 4.79\left(\mathrm{~s}, 2 \mathrm{H}, \mathrm{CH}_{2}\right)\end{array}$ \\
\hline
\end{tabular}

\section{References}

1. Menon M K, Clark W G and Ures D A, Eur J Pharmacol.,1972, 19, 43-51.

2. $\quad$ Pandey V K and Agarwat A K, J Indian Chem Soc., 1981, 58, 306-307.

3. Spasov A A, Yozhitsa I N, Bugaeva L I and Anisimova V A, Pharm Chem J., 1999, 33, 232-243.

4. a) Gravatt G L, Baguley B C, Wilson W R and Denny W A, J Med Chem., 1994, 37, 4338-4345;. b) Jayashankara B and Rai K M L, ARKIVOC, 2008, 11, 75.

5. El-Ablack F Z, Etmann H A, Metwally M A and Amer F A, Pharmzie, 1995, 50(3), 222-224.

6. El-Ablack F Z, Etman H A and Metwally M A, Boll Chim Farmaceutico.,1995, 134, 77-79.

7. Mohareb R M, Wardakhan W W and El-Ablack F Z, J Chem Res (M), 1994, 747-760.

8. El-Ablack F Z, Boll Chi Farmac., 2003, 9, 142.

9. Reddy B S, Reddy R B, Chandramouli G V P and Reddy Y D, J Indian Chem Soc., 1988, 65, 853-854. 


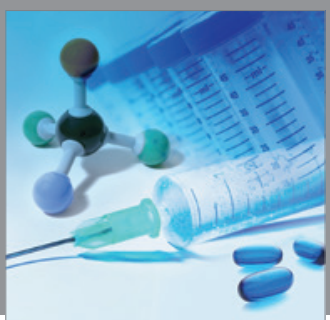

International Journal of

Medicinal Chemistry

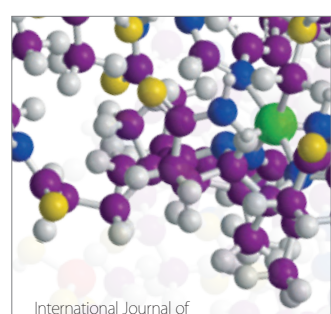

Carbohydrate Chemistry

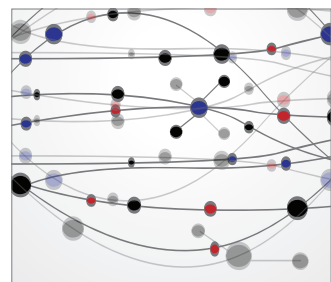

The Scientific World Journal
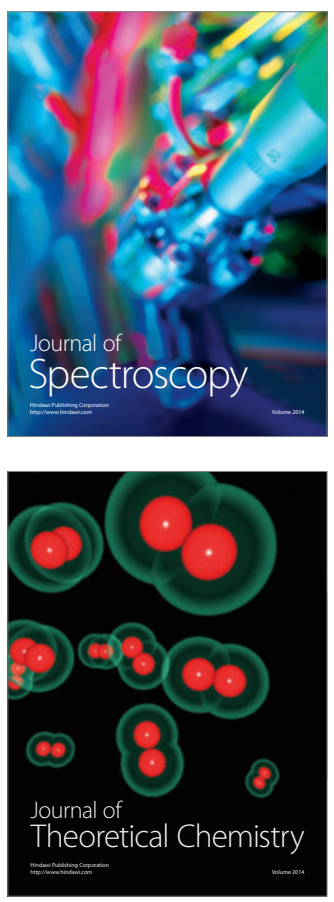
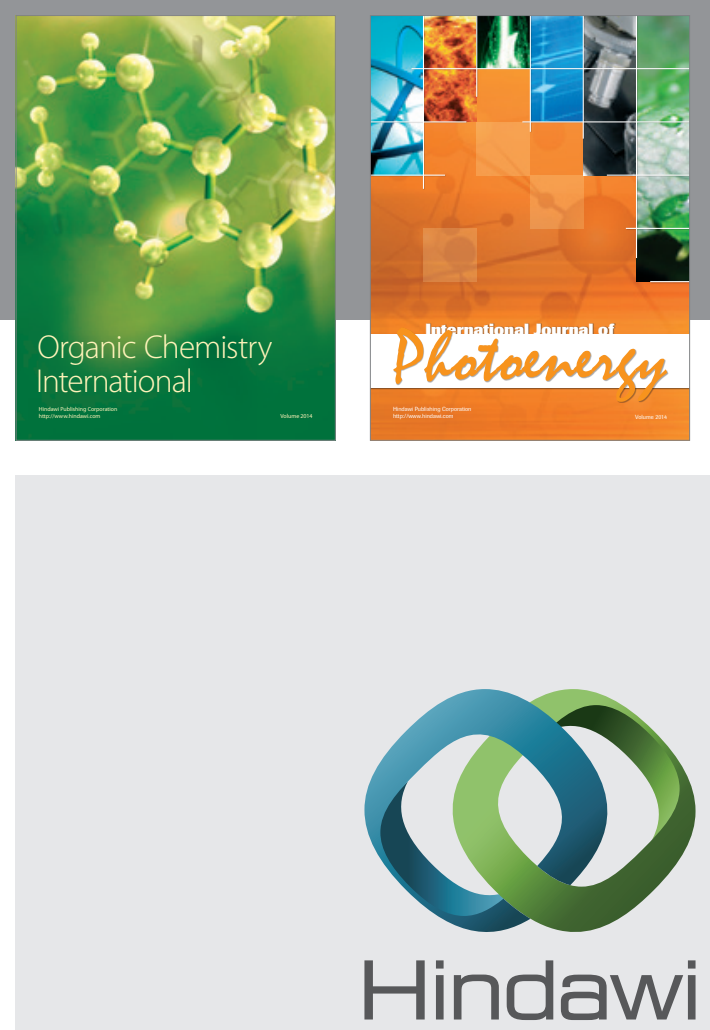

Submit your manuscripts at

http://www.hindawi.com
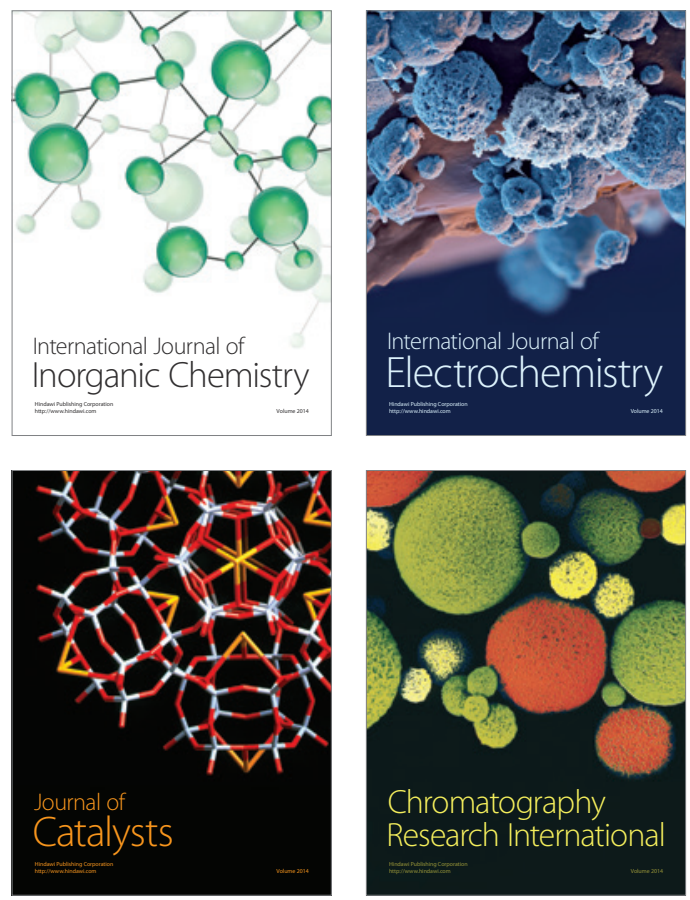
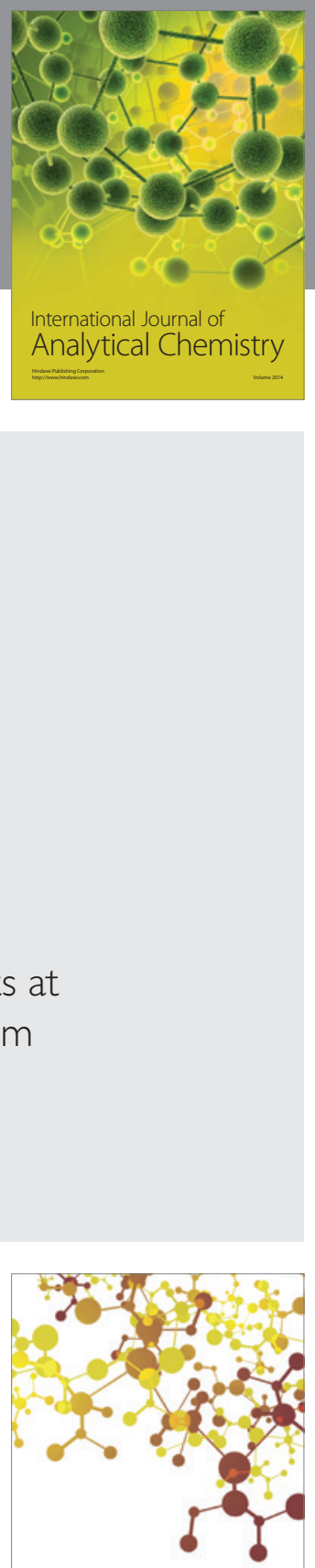

Journal of

Applied Chemistry
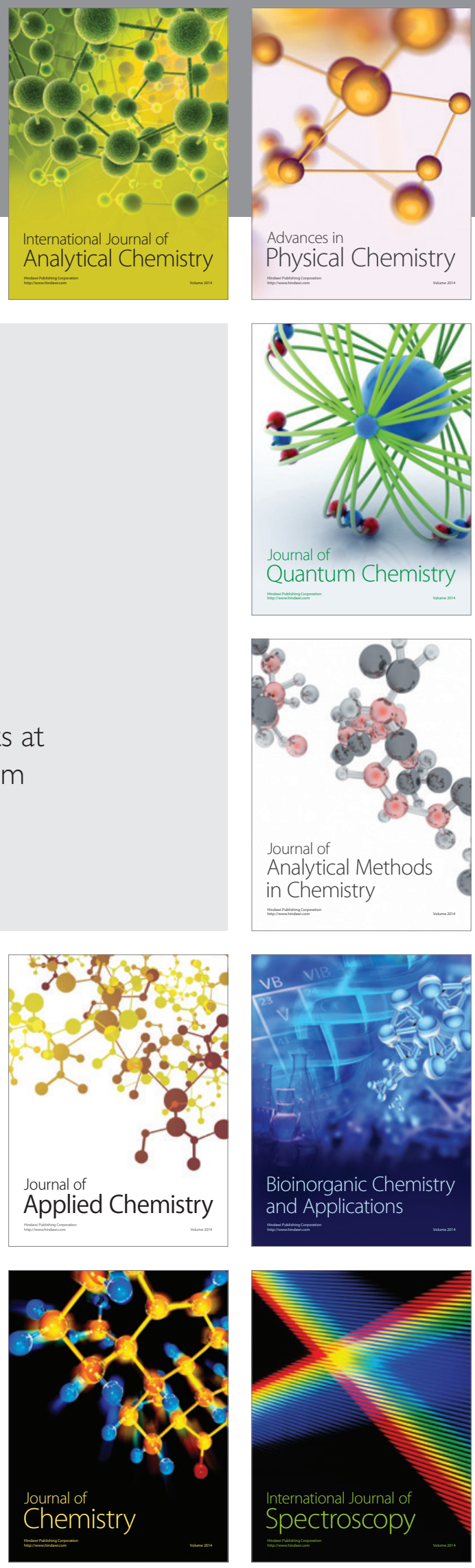